\title{
Die Erfolge staatlicher Förderung
}

\author{
Die Niederlande streben mit ihrem Umwelt-Unternehmens-Plan konkrete \\ Umweltziele an. Dazu gehören auch Veränderungen, die in enger Zusammenar- \\ beit mit der Industrie durchgeführt werden müssen. Die bisherigen Ergebnisse \\ zeigen, daß eine Stärkung des Umweltmanagements in den Unternehmen durch \\ eine sinnvolle Zusammenarbeit mit den Behörden ergänzt werden muß.
}

M it dem niederländischen Umweltplan genannt „National Environmental Policy Plan“ (NEPP) soll eine nachhaltige Gesellschaft innerhalb des Zeitraums einer Generation verwirklicht werden. Dabei setzen die Niederländer insbesondere auf die sogenannte industrielle Zielgruppenpolitik (,Target Group Policy for Industry"). Diese überträgt die Ziele des niederländischen Umweltplanes in Ziele für die Gesamtindustrie, die dann weiter auf-gesplittet werden in Umweltziele für Industriebranchen und schließlich für einzelne Unternehmen. Dem liegt eine von Industrie und staatlichen Behörden unterzeichnete Absichtserklärung zugrunde. Große und komplexere Unternehmen müssen in diesem Rahmen ihren eigenen betrieblichen Umweltplan, „Company Environmental Plan" (CEP) entwerfen. Aufgabe der Behörden ist es, diesen CEP zu überprïfen und zu genehmigen. Der CEP bildet dann die Grundlage fuir weitere behördliche Genehmigungen und Überwachungen. Kleine und mittlere Unternehmen fiillen eine Art Checkliste aus.

\section{- Umweltmanagement}

Durch die industrielle Zielgruppenpolitik und die betrieblichen Umweltpläne ist geregelt, was die Unternehmen zu tun haben. Darüber hinaus benötigen sie aber auch ein Umweltmanagementsystem als das Instrument zur Verwirklichung einer nachhaltiger Entwicklung. 1989 setzte die niederländische Regierung das Ziel fest, daß bis 1995 nahezu alle 10.000 holländischen Unternehmen, die die Umwelt mäßig bis schwer belasten oder spezifische Risiken darstellen, ein Umweltmanagementsystem etabliert haben sollten, allerdings auf freiwilliger Basis. Von den übrigen, die Umwelt nur begrenzt belastenden 250.000 Unternehmen Industriebranchen, wurde und wird erwartet, daß auch sie Schritte zur Einführung eines Umweltmanagementsystems unternehmen.
Um die Implementierung von Umweltmanagementsystemen voranzutreiben, stellte der Staat während eines Zeitraums von fünf Jahren 50 Millionen holländischer Gulden für etwa 225 Projekte in 65 Industriebranchen zur Verfügung. Auch wurden 25 ,Industrial Environmental Services“ (Umweltberatungsstellen) eingerichtet.

\section{- Numerische Ziele weniger wichtig}

Die Entwicklung des Umweltmanagements wurde 1990 und 1993 begutachtet. Bei der Gruppe der 10.000 mäßig bis stark umweltbelastenden Unternehmen waren deutliche Fortschritte erkennbar, und zwar sowohl bei großen als auch bei kleineren Unternehmen. Allerdings zeichnete sich bei der Zwischenbilanz von 1993 auch ab, daß die Ziele für 1995 nur teilweise erreicht werden können. Vor diesem Hintergrund entschied die niederländische Regierung, es sei weniger wichtig, numerische Ziele zu erreichen, als Umweltmanagementsysteme in den Unternehmen auf eine solide Grundlage zu stellen. Der Prozess sollte aber gleichzeitig beschleunigt werden. Deshalb hat die niederländische Regierung die Schwerpunkte dieser Politik seit 1994 anders festgelegt. Diese sind heute: Genehmigung und Überwachung, industrielle Zielgruppenpolitik, Normierung und Zertifizierung, eine gesetzliche Verpflichtung zur Umweltberichterstattung und eine Erweiterung der Überwachungsinstrumente um ein Umwelt-Audit.

Darüber hinaus sollten staatliche Eingriffe auf die Haltung der Unternehmen zu Umweltfragen abgestimmt werden. Für die führenden Unternehmen ist es heute sehr wichtig, daß zwischen Genehmigung und Überwachung von Anlagen und Umweltmanagementsystemen ein Zusammenhang besteht. Hier bedarf es auch einer engen Abstimmung der Zertifizierung von Umweltmanagementsystemen mit der Teilnahme am EG-Öko-Audit-System.
Gegenwärtig werden Normierung und Zertifizierung von Umweltmanagementsystemen in den Niederlanden auf der Basis der britischen Umweltmanagementnorm BS 7750 und nach dem EG-Öko-Audit-System implementiert: Es gibt dabei nur eine einzige Struktur dem ein zentrales Zertifizierungsund Verifizierungsschema zu Grunde liegt, einen Sachverständigenrat (,Central Council of Experts“) und eine Prüfungsstelle (,EMS Certification Coordination Council Office"). Dieses Amt ist gleichzeitig offiziell für die Umsetzung des EG-Öko-Audit zuständig. Zertifizierung und Berichterstattung sind auf diese Art Kommunikationsbasis der Beziehungen zwischen Regierung und Unternehmen.

\section{Anpassung des Ordnungsrechts}

Wenn eine Firma eigenverantwortlich einen betrieblichen Umweltplan aufstellt, ein zertifiziertes Umweltmanagementsystem hat und über ihre Umweltschutzleistung öffentlich berichtet, dann ist es sinnvoll, Umweltmanagement und Ordnungsrecht aufeinander abzustimmen. Deshalb ist es in den Niederlanden möglich, eine sogenannte Rahmengenehmigung (,outline licence") zu erhalten, die sich nur auf die Ziele des Unternehmens als Ganzem bezieht; und auf der Grundlage der vom Umweltmanagementsystem gelieferten Informationen überwacht. Wichtig ist aber, daß eine kontinuierliche Verbesserung der Umweltschutzleistung angestrebt wird. Insgesamt schafft eine derartige wirkungsvolle Ergänzung zwischen Behörden und Unternehmen eine viel größere Flexibilität.

In diesem Sinn geht es der niederländischen Politik heute hauptsächlich darum, eine andere Beziehung zwischen Unternehmen und Behörden zu schaffen, insbesondere im Hinblick auf lokale Behörden. Die Schlüsselbegriffe hierfür sind: unternehmerische Eigenverantwortung, be-trieblicher Umweltplan, laufende Verbesserungen, Zertifizierung, Umweltberichterstattung und die Rahmenzulassung. Diese werden sich auch nach der diesjährigen Zwischenbilanz nicht wesentlich ändern.

\section{Der Referent}

Hugo H. Kuijier ist Koordinator für Umweltmanage ment in der Abteilung Industrie (Division Industry) des niederländischen Umweltministeriums, Den Haag

Kontakt: Tel. $(++31)(70)$ 339-4063, Fax -1293 
(c) 20I0 Authors; licensee IÖW and oekom verlag. This is an article distributed under the terms of the Creative Commons Attribution Non-Commercial No Derivates License (http://creativecommons.org/licenses/by-nc-nd/3.o/), which permits unrestricted use, distribution, and reproduction in any medium, provided the original work is properly cited. 